\title{
Talla de madurez y época de desove de la reineta (Brama australis Valenciennes, 1836) en la costa central de Chile
}

\author{
Elson Leal \& Ciro Oyarzún \\ Departamento de Oceanografía, Universidad de Concepción \\ Casilla 160-C, Concepción, Chile \\ E-mail: eleal@udec.cl; coyarzun@udec.cl
}

RESUMEN. La reineta (Brama australis) adquiere cada vez mayor importancia en los desembarques de la pesca artesanal, no obstante, es escasa la información que existe acerca de su biología básica, particularmente sobre su dinámica reproductiva. En este trabajo se determina la época principal de desove de B. australis en la costa central de Chile y la talla de madurez (TM), para lo cual se analizó un total de 1061 individuos capturados frente a Lebu (37²37'S$73^{\circ} 40^{\prime} \mathrm{W}$ ), entre enero y septiembre de 2001. El análisis macroscópico de los ovarios, mostró ejemplares sexualmente maduros durante todo el período de muestreo. El Indice Gonadosomático (IGS) presentó fluctuaciones que indican un extenso período de desove, con un máximo invernal (junio-agosto). La TM se calculó a partir del método de longitud del $50 \%$ de hembras maduras a los $36,9 \mathrm{~cm}$ longitud horquilla (LH), con intervalo de confianza entre 35,3 y $37,8 \mathrm{~cm}$ y en $39 \mathrm{~cm}$ LH mediante el método del Incremento Relativo del IGS (IR.IGS). Debido a que las capturas ocurrieron principalmente en ejemplares sobre $39 \mathrm{~cm} \mathrm{LH}$, la flota estaría extrayendo mayoritariamente peces maduros.

Palabras claves: reineta, talla de madurez, desove, Brama, Chile.

\section{Maturity size and spawning time of the Pacific pomfret (Brama australis Valenciennes, 1836) in the central coast of Chile}

\begin{abstract}
Landings of Pacific pomfret (Brama australis) is acquiring an increasing importance to the Chilean artisanal fishery. However, lack of information regarding its basic biology, in particular, reproductive dynamics, still persists. In this work we determine the spawning season of $B$. australis in the central coast of Chile and the length of maturity (LM). A total of 1061 individuals captured in the coastal zone of Lebu (37 $\left.37^{\prime} \mathrm{S}-73^{\circ} 40^{\prime} \mathrm{W}\right)$, Chile, between January and September 2001, were analyzed. Macroscopic analysis of ovaries, showed sexually mature individuals throughout the sampling period. Gonadosomatic Index (IGS) showed fluctuations indicative of extensive spawning periods, with a maximum in the austral winter season (June-August). LM of females was estimated with the length at $50 \%$ maturity method, in $36.9 \mathrm{~cm}$ fork length (LH), with a confidence interval between 35.3 and $37.8 \mathrm{~cm} \mathrm{LH}$, and 39 $\mathrm{cm}$ LH using the Relative Increment of IGS method (IR.IGS). Since the catch comprises principally individuals over $39 \mathrm{~cm} \mathrm{LH}$, the fishing fleet extracts mainly mature fish.
\end{abstract}

Key words: Pacific pomfret, spawning, mean maturity size, Brama, Chile.

\section{INTRODUCCIÓN}

Brama australis (Bramidae: Perciformes) es conocida en Chile con los nombres comunes de reineta, pez hacha o palometa del sur, e internacionalmente como Pacific pomfret. Según Pavlov (1991a) esta especie se distribuye dentro de los límites de circulación de masas de agua subtropicales. En Chile se distribuye desde Coquimbo $\left(29^{\circ} \mathrm{S}\right)$ hasta el extremo sur-austral (Oyarzún, 2001), siendo capturada principalmente con espineles por pescadores artesanales en aguas costeras, apareciendo además, como fauna acompañante en la pesquería industrial de cerco. Esta especie se registró por primera vez en el Anua- 
rio Estadístico de Pesca de Chile en 1994, con 1.186 ton desembarcadas principalmente en Valparaíso ( $\left.33^{\circ} \mathrm{S}-71^{\circ} 50^{\prime} \mathrm{W}\right)$. Sin embargo, en 1999 y 2000 más del $50 \%$ de las capturas se desembarcaron en Lebu, las que el 2001 superaron las 15.000 ton (Fig. 1).

La importancia adquirida por B. australis en los desembarques de la pesca artesanal, no ha estado acompañada de estudios suficientes, que den cuenta de aspectos básicos de su ciclo de vida. El conocimiento acerca de su biología es mínimo, desconociendo aspectos importantes, como la reproducción. Esto es más destacado aún, dado que se trata de una especie de relevancia económica potencial o actual.

Los antecedentes sobre los aspectos reproductivos de B. australis indican que es una especie con desove parcial con su máximo de actividad reproductiva de abril a junio (Pavez et al., 1998). La talla media de madurez es de $35 \mathrm{~cm}$ de longitud de horquilla (Pavlov, 1991b), no registrándose información adicional sobre este parámetro. En el presente estudio se analizan algunos aspectos reproductivos importantes de $B$. australis frente a la costa central de Chile, determinando la talla de madurez de las hembras y su época principal de desove.

\section{MATERIALES Y MÉTODOS}

El área de estudio se ubicó frente a la costa de Lebu ( $37^{\circ} 37^{\prime}$ S- $-73^{\circ} 40^{\prime} \mathrm{W}$ ), Chile (Fig. 2). Las muestras se obtuvieron de la flota artesanal espinelera que operó en esta zona, cercana a la costa. Se realizaron muestreos mensuales de $B$. australis en el puerto pesquero artesanal de Lebu desde enero hasta septiembre de 2001. Cada pez fue sexado, pesado y medido en fresco en su longitud de horquilla (LH). Tanto a hembras como a machos se les extrajeron las gónadas y se pesaron. Además, se registró el estado de madurez (EM) de las hembras mediante la escala macroscópica de cinco estados, elaborada para desovantes parciales por Holden \& Raitt (1975).

El muestreo se realizó en forma semidirigida sobre las hembras. Es decir, una vez que los ejemplares se sexaron, los machos se muestrearon ocasionalmente (sólo para analizar la variación mensual del IGS), mientras que el mayor tamaño de muestra se obtuvo de las hembras, ya que con ellas se estimó la talla de madurez (TM).

La época principal de desove se determinó usando el Indice Gonadosomático (IGS) (Nikolsky, 1963), estimando un promedio mensual de su valor para machos y hembras. El IGS, se expresó como:

$$
\text { IGS }=\frac{\mathrm{P}_{\mathrm{g}}}{\left(\mathrm{P}_{\mathrm{t}}-\mathrm{P}_{\mathrm{g}}\right)} \cdot 100
$$

donde:

$$
\begin{aligned}
& \mathrm{P}_{\mathrm{g}}=\text { peso de la gónada }(\mathrm{g}) \\
& \mathrm{P}_{\mathrm{t}}=\text { peso total del pez }(\mathrm{g})
\end{aligned}
$$

La TM se determinó mediante el método del Incremento Relativo del Indice Gonadosomático (IR.IGS) (Alarcón \& Arancibia, 1993; Arancibia et al., 1994). Las hembras se agruparon arbitrariamente en clases de tamaño a cada centímetro de longitud

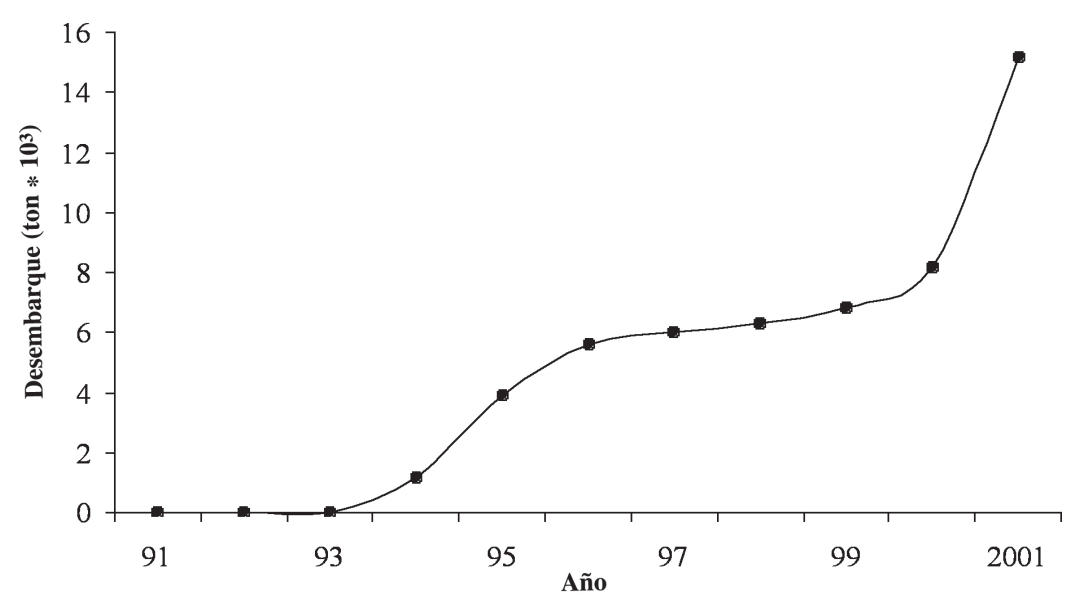

Figura 1. Desembarque anual de reineta en Chile desde 1991 a 2001 (Servicio Nacional de Pesca, 2001).

Figure 1. Annual landing of Pacific pomfret in Chile from 1991 to 2001 (Servicio Nacional de Pesca, 2001). 
horquilla, siendo consideradas sólo las muestras de junio, julio y agosto. La expresión que describió el IR.IGS fue:

$$
\text { IR.IGS }=\frac{\left(\mathrm{IGS}_{\mathrm{j}+1}-\mathrm{IGS}_{\mathrm{j}}\right)}{\mathrm{IGS}_{\mathrm{j}}} \cdot 100
$$

donde:

$\begin{aligned} \text { IGS }_{\mathrm{j}+1}= & \text { corresponde al IGS promedio de la clase } \\ & \text { de tamaño } \mathrm{j}+1\end{aligned}$

$\mathrm{IGS}_{\mathrm{j}}=$ es el IGS promedio de la clase de tamaño $\mathrm{j}$

Además, la TM se estimó como la longitud a la cual el $50 \%$ de hembras están maduras $\left(1_{50 \%}\right)$ (Pardo \& Oliva, 1992; Alarcón \& Arancibia, 1993; Arancibia et al., 1994), para lo cual se consideraron como maduras aquellas hembras cuyas gónadas se encontraron en estados III y IV. La expresión utilizada es una función de tipo logística, cuya forma es:

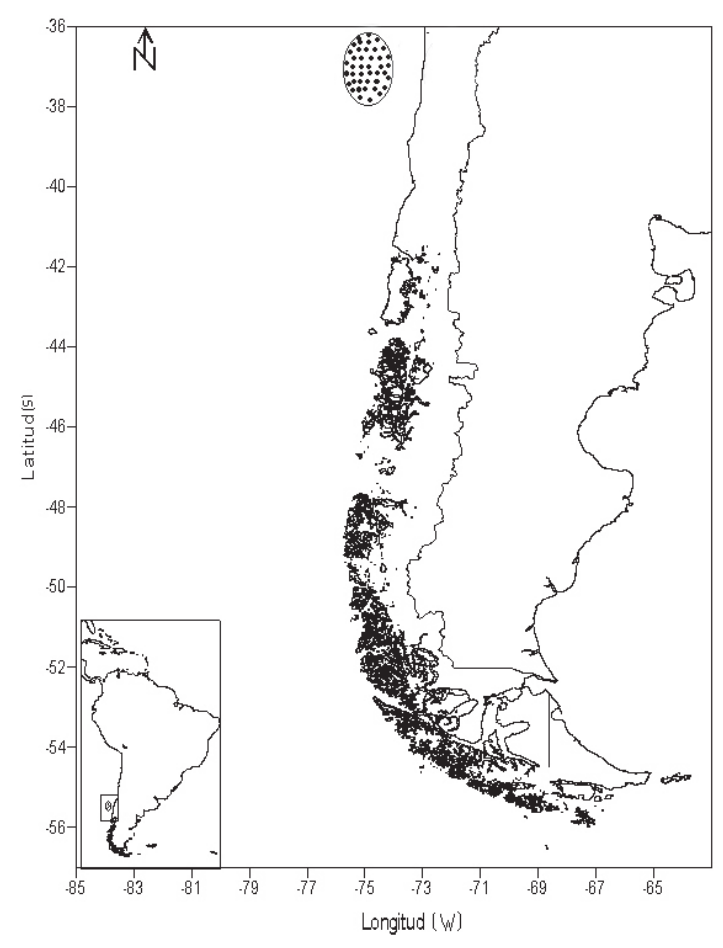

Figura 2. Zona de pesca de la reineta desembarcada en el puerto de Lebu durante el periodo de muestreo. Figure 2. Fishing zone of Pacific pomfret, landed in Lebu harbour during the sampling period.

$$
\mathrm{P}_{\mathrm{LH}}=\frac{1}{\left(1+\mathrm{e}^{\left(\beta_{0}+\beta_{1} * \mathrm{Lh}\right)}\right)}
$$

donde:

$\mathrm{P}_{\mathrm{LH}} \quad=$ corresponde a la proporción de hembras maduras a la longitud de horquilla (LH).

$\beta_{0}$ y $\beta_{1}=$ son constantes del modelo.

Se estimó el intervalo de confianza (IC) para la longitud de $50 \%$ de individuos maduros $\left(\mathrm{P}_{0.5}\right)$. Para lo cual se realizó un remuestreo tipo Monte Carlo (con el algoritmo MATSIM) de los parámetros de madurez $\beta_{0}$ y $\beta_{1}$, generando de esta forma la distribución de probabilidad del estadístico $\mathrm{P}_{0.5}$ derivado (Roa et al., 1999). Los parámetros $\beta_{0}$ y $\beta_{1}$ se obtuvieron por Máxima Verosimilitud mediante el programa estadístico SYSTAT, considerando una distribución binomial de los datos de madurez. Luego, la proporción de 0,5 individuos maduros se calculó mediante la siguiente ecuación:

$$
P_{0,5}=-\frac{\beta_{0}}{\beta_{1}}
$$

\section{RESULTADOS}

Se analizó un total de 1061 individuos (673 hembras y 388 machos). La longitud de los ejemplares fluctuó entre 27 y $50 \mathrm{~cm}$ LH en hembras y, entre 30 y $51 \mathrm{~cm} \mathrm{LH}$ en machos. La talla promedio mensual de los ejemplares analizados varió entre 38,5 y 40,6 cm LH, y entre 38,7 y 44,4 cm LH, respectivamente (Tabla 1).

Los mayores valores promedio del IGS en ambos sexos se presentaron en junio, julio y agosto (Tabla 2), indicando una alta actividad reproductiva en el período invernal. La variación mensual del IGS en ambos sexos fue similar durante todos los meses en que se realizó el muestreo (Fig. 3), evidenciando un sincronismo en su maduración gonadal.

El número de hembras examinadas durante el período de máxima actividad reproductiva a las cuales se aplicaron los criterios de madurez, se indican en la Tabla 3. Se observaron ejemplares con ovario maduro (EM III y IV) durante todos los meses considerados, indicando que este recurso es un desovante parcial. Sin embargo, no se encontraron hembras en estado de post-desove (EM V).

La TM, de $B$. australis, calculada mediante el método del IR.IGS correspondió a 39 cm LH (Fig. 
Tabla 1. Tallas promedio, mínimas y máxima (cm LH) de machos y hembras de B. australis durante el período de muestreo.

Table 1. Average, minimum and maximum sizes (cm LH) of males and females of $B$. australis during the sampling period.

\begin{tabular}{|ccccccc|}
\hline Mes & Talla & $\begin{array}{c}\text { Hembras } \\
\text { Talla } \\
\text { mínima }\end{array}$ & $\begin{array}{c}\text { Talla } \\
\text { promedio }\end{array}$ & $\begin{array}{c}\text { Talla } \\
\text { máxima }\end{array}$ & $\begin{array}{c}\text { Talla } \\
\text { mínima }\end{array}$ & $\begin{array}{c}\text { Talla } \\
\text { promedio }\end{array}$ \\
máxima \\
\hline Enero & 27,0 & 38,9 & 43,0 & 42,0 & 44,4 & 51,0 \\
Febrero & 29,5 & 39,9 & 44,5 & 36,0 & 41,9 & 44,5 \\
Marzo & 28,5 & 38,5 & 47,5 & 33,5 & 39,3 & 49,0 \\
Abril & 34,0 & 39,9 & 47,0 & 29,0 & 40,4 & 48,0 \\
Mayo & 36,5 & 39,6 & 45,0 & 34,5 & 38,7 & 43,0 \\
Junio & 30,0 & 40,3 & 49,0 & 30,0 & 39,5 & 46,5 \\
Julio & 27,5 & 40,6 & 47,5 & 32,0 & 42,1 & 49,0 \\
Agosto & 34,0 & 40,6 & 50,0 & 38,0 & 42,1 & 46,5 \\
Septiembre & 35,0 & 38,8 & 45,0 & 36,0 & 39,5 & 44,0 \\
\hline
\end{tabular}

Tabla 2. Promedio mensual y desviación estándar del IGS de machos y hembras de B australis, durante el periodo de muestreo.

Table 2. Monthly IGS average and standard deviation in males and females of $\boldsymbol{B}$. australis during the sampling period.

\begin{tabular}{|c|c|c|c|c|c|c|c|}
\hline \multirow[t]{2}{*}{ Mes } & \multicolumn{3}{|c|}{ Hembras } & \multicolumn{3}{|c|}{ Machos } & \multirow[t]{2}{*}{ Total } \\
\hline & $\begin{array}{l}\text { Promedio } \\
\text { IGS }\end{array}$ & $\begin{array}{l}\text { Desviación } \\
\text { estándar }\end{array}$ & $\mathbf{n}$ & $\begin{array}{l}\text { Promedio } \\
\text { IGS }\end{array}$ & $\begin{array}{c}\text { Desviación } \\
\text { estándar }\end{array}$ & $\mathbf{n}$ & \\
\hline Enero & 1,08 & 0,48 & 81 & 0,23 & 0,012 & 43 & 124 \\
\hline Febrero & 1,05 & 0,43 & 100 & 0,24 & 0,12 & 32 & 132 \\
\hline Marzo & 0,83 & 0,38 & 104 & 0,17 & 0,09 & 40 & 144 \\
\hline Abril & 1,00 & 0,43 & 80 & 0,23 & 0,15 & 71 & 151 \\
\hline Mayo & 0,85 & 0,37 & 26 & 0,15 & 0,08 & 14 & 40 \\
\hline Junio & 1,26 & 0,53 & 40 & 0,19 & 0,07 & 35 & 75 \\
\hline Julio & 1,36 & 0,63 & 149 & 0,28 & 0,14 & 97 & 246 \\
\hline Agosto & 1,47 & 0,72 & 49 & 0,27 & 0,12 & 16 & 65 \\
\hline Septiembre & 0,94 & 0,47 & 44 & 0,22 & 0,09 & 40 & 84 \\
\hline Total & & & 673 & & & 388 & 1061 \\
\hline
\end{tabular}

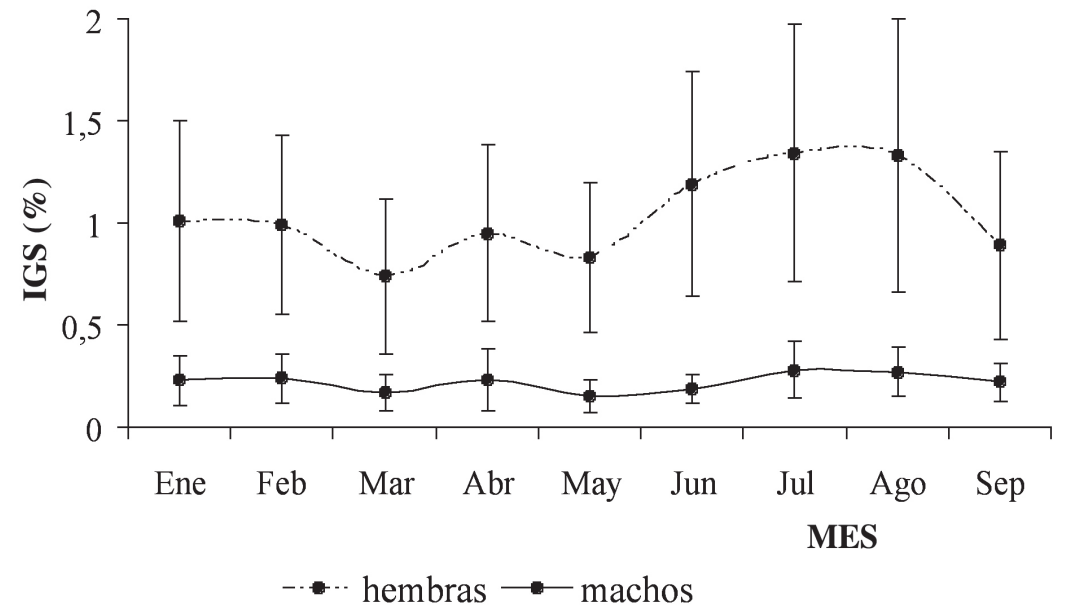

Figura 3. Promedio mensual y desviación estándar del Indice Gonadosomático para machos y hembras de $B$. australis analizados durante el período eneroseptiembre de 2001.

Figure 3. Monthly average and standard deviation of the Gonadosomatic Index for males and females of $\boldsymbol{B}$. australis analyzed during January-September period of 2001. 
Tabla 3. Frecuencia de hembras de B. australis examinadas, por estado de madurez (EM), entre enero y septiembre de 2001.

Table 3. Females frequency of B. australis examined for each maturity stage (ME) between January and September 2001.

\begin{tabular}{|crrrrrrrrrr|r|}
\hline EM & Enero & Febrero & Marzo & Abril & Mayo & Junio & Julio & Agosto & Septiembre & Total \\
\hline I & 9 & 15 & 27 & 11 & 1 & 2 & 17 & 2 & 1 & 85 \\
II & 17 & 20 & 26 & 12 & 8 & 5 & 14 & 7 & 20 & 129 \\
III & 10 & 9 & 25 & 13 & 8 & 7 & 27 & 5 & 9 & 113 \\
IV & 45 & 56 & 26 & 44 & 9 & 26 & 91 & 35 & 14 & 346 \\
V & 0 & 0 & 0 & 0 & 0 & 0 & 0 & 0 & 0 & 0 \\
Total & 81 & 100 & 104 & 80 & 26 & 40 & 1149 & 49 & 44 & 673 \\
\hline
\end{tabular}

Tabla 4. Proporción observada y esperada de hembras maduras de $B$. australis por clase de longitud, analizadas durante la época de mayor actividad reproductiva (período junio-agosto).

Table 4. Observed and expected mature female proportion of $\boldsymbol{B}$. australis by length class, analyzed during the period of most reproductive activity (JuneAugust period).

\begin{tabular}{|ccccc|}
\hline $\begin{array}{c}\text { LH } \\
\text { (cm) }\end{array}$ & $\begin{array}{c}\text { Frecuencia } \\
\text { inmaduro }\end{array}$ & $\begin{array}{c}\text { Frecuencia } \\
\text { maduro }\end{array}$ & $\begin{array}{c}\text { Proporción } \\
\text { observada }\end{array}$ & $\begin{array}{c}\text { Proporción } \\
\text { esperada }\end{array}$ \\
\hline 27 & 0 & 0 & 0,00 & 0,00 \\
28 & 1 & 0 & 0,00 & 0,00 \\
29 & 0 & 0 & 0,00 & 0,00 \\
30 & 5 & 0 & 0,00 & 0,01 \\
31 & 3 & 0 & 0,00 & 0,01 \\
32 & 5 & 0 & 0,00 & 0,03 \\
33 & 3 & 0 & 0,00 & 0,06 \\
34 & 2 & 0 & 0,00 & 0,11 \\
35 & 1 & 0 & 0,00 & 0,21 \\
36 & 7 & 3 & 0,30 & 0,35 \\
37 & 6 & 11 & 0,65 & 0,53 \\
38 & 5 & 16 & 0,76 & 0,70 \\
39 & 5 & 11 & 0,69 & 0,82 \\
40 & 2 & 16 & 0,89 & 0,91 \\
41 & 1 & 26 & 0,96 & 0,95 \\
42 & 0 & 26 & 1,00 & 0,98 \\
43 & 0 & 21 & 1,00 & 0,99 \\
44 & 1 & 23 & 0,96 & 0,99 \\
45 & 0 & 20 & 1,00 & 1,00 \\
46 & 0 & 12 & 1,00 & 1,00 \\
47 & 0 & 7 & 1,00 & 1,00 \\
48 & 0 & 1 & 1,00 & 1,00 \\
49 & 0 & 1 & 1,00 & 1,00 \\
50 & 0 & 1 & 1,00 & 1,00 \\
& & & & \\
\hline
\end{tabular}

4), con un incremento relativo de $31,2 \%$. A su vez, al utilizar el criterio del $50 \%$ de hembras maduras $\left(\mathrm{L}_{50 \%}\right)$ este parámetro fue menor, estimándose en $36,9 \mathrm{~cm}$ de $\mathrm{LH}$ con valores para $\beta_{0}$ y $\beta_{1}$ de 26,59 y $-0,72$, respectivamente. Se estimó un IC del 95\% para $\mathrm{L}_{50 \%}$ entre 35,3 y $37,8 \mathrm{~cm}$ con valores de $\beta_{0}$ entre 20,34 y 23,31 , y $\beta_{1}$ entre $-0,58$ y -0,62. La proporción observada de hembras maduras por LH y la proporción estimada, se muestra en la Figura 5. La función de madurez establecida para esta especie es la siguiente:

$$
\mathrm{P}_{\mathrm{LH}}=\frac{1}{\left(1+\mathrm{e}^{(-0,58 * \mathrm{Lh})}\right)}
$$

\section{DISCUSIÓN}

La curva de madurez expresada en el IGS, así como la presencia de hembras maduras (EM III y IV) durante todos los meses de muestreo, indican que la reineta (B. australis) en la zona centro sur de Chile es un desovante parcial o fraccionado, con un período prolongado de madurez gonádica y una época principal de desove en invierno (junio-agosto). Este resultado coincide parcialmente con lo señalado por Pavez et al. (1998), quienes señalan que en la zona de Valparaíso, B. australis tiene un desove parcial durante el año, con un máximo reproductivo entre abril y junio, que corresponde al periodo de otoñoinvierno en el hemisferio sur. Sin embargo, estos resultados discrepan con Pavlov (1991b), quien reporta la actividad principal de desove de esta especie durante primavera-verano, al estudiar individuos capturados en aguas oceánicas de la zona sur-austral (42 $59^{\prime}-47^{\circ} 01^{\prime} \mathrm{S}$; $\left.107^{\circ} 19^{\prime}-126^{\circ} 58^{\prime} \mathrm{W}\right)$.

Otro representante de la Familia Bramidae ( $\mathrm{Bra}$ ma japonica) es un desovante invernal en el 

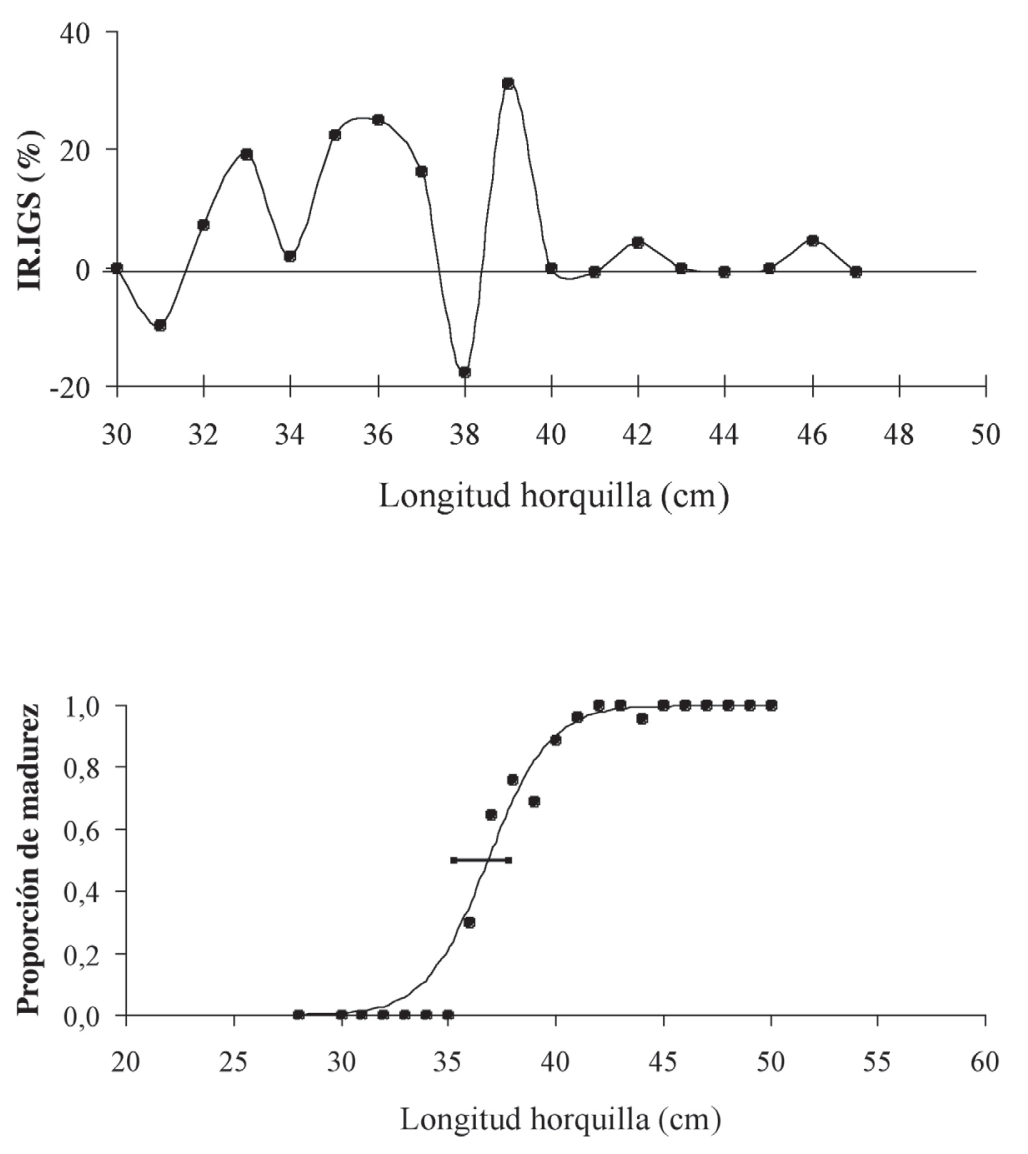

Figura 4. Talla de madurez estimada para hembras de $B$. australis, a través del criterio del Incremento Relativo del IGS.

Figure 4. Maturity size estimated for female of $B$. australis using the criterion of Relative Increment of the IGS.

Figura 5. Talla de madurez de $B$. australis, estimada a través del criterio de $50 \%$ de hembras maduras, $(\bullet)$ datos observados, (-) modelo ajustado, $(\bullet-\bullet)$ intervalo de confianza para $\mathrm{L}_{\mathbf{5 0}} \%$

Figure 5. Maturity size of $B$. australis estimated with the $50 \%$ mature females criterion, $(\bullet)$ observed data, (-) fitted model, $(\bullet-\bullet)$ confidence interval for $\mathbf{L}_{\mathbf{5 0} \%}$ subtrópico del Pacífico norte $\left(25^{\circ}\right.$ a $\left.50^{\circ} \mathrm{N}\right)$ (Shimazaki \& Nakamura 1981, fide William et al., 1993). Esto entrega antecedentes a favor del desove principal de B. australis durante el invierno en nuestras costas, cuya distribución en el hemisferio sur $\left(20^{\circ}\right.$ a $\left.40^{\circ} \mathrm{S}\right)$ coincide con la de B. japonica en el Pacífico norte.

Cubillos et al. (1999) estudiando aspectos reproductivos de Strangomera benticki en el sistema de surgencia de Chile centro-sur, plantean que el desove de esta especie a fines de invierno (agosto), sería una estrategia adaptativa de la población para que los estados larvales encuentren condiciones favorables para su sobrevivencia al inicio de la primavera (septiembre), cuando la surgencia costera es moderada. En la costa central de Chile $\left(35^{\circ}-\right.$ $\left.40^{\circ} \mathrm{S}\right)$, los eventos de surgencia en septiembre se caracterizan por ser moderados (Arcos et al., 1996), lo cual debería favorecer la disponibilidad de alimento para las larvas de B. australis, posterior al desove.
Castro et al. (2000), amplían la hipótesis de áreas de retención larval, planteada por Parrish et al. (1981) para el desove invernal de anchoveta (Engraulis ringens) en la zona central de Chile, explicando que durante el invierno, los vientos provenientes del norte, producen un transporte superficial de aguas en dirección a la costa, donde existirían condiciones más adecuadas para el desarrollo de las larvas.

El mayor valor de IGS encontrado en agosto en B. australis se interpreta como un máximo reproductivo durante este mes, que permite postular la hipótesis que el desove invernal podría responder a una estrategia del recurso, para aumentar la probabilidad de sobrevivencia de las larvas, las cuales encontrarían condiciones oceanográficas y de alimentación adecuadas. No obstante, para su confirmación es necesario realizar estudios sobre otros aspectos reproductivos de $B$. australis, como la variación de abundancia y distribución de las larvas de esta especie. 
La ausencia de hembras en estado de post-desove se puede explicar ya sea por la corta duración de este estado o por que el proceso de desove se produce en áreas más alejadas de la costa, en comparación con la zona donde opera la flota pesquera artesanal.

La TM estimada según el IR.IGS en hembras de B. australis, en $39 \mathrm{~cm} \mathrm{LH}$, con una variación del $31,2 \%$ (Fig. 4), no resultó en un incremento significativamente alto del IGS, como se espera a una cierta longitud, en la cual se evidencia un proceso claro de madurez. Por ejemplo, Alarcón \& Arancibia (1993) al estudiar la madurez en merluza común (Meluccius gayi gayi), encontraron una variación de alrededor de $200 \%$ en el IGS promedio entre intervalos de longitud, mientras que Arancibia et al. (1994), informaron un IR.IGS de $109 \%$ para la talla de madurez en sardina común (Strangomera bentincki). Sin embargo, la TM estimada a partir de este método es similar a la informada para $B$. australis por Pavez et al. (1998), quienes indican que la TM estaría entre 36,9 y 38,9 cm LH.

La TM estimada según el criterio de $50 \%$ de hembras maduras en 36,9 cm LH (IC 35,3-37,8), coincide con lo señalado por Pavez et al. (1998) y reafirma además, lo publicado por Pavlov (1991b), quien indica que esta especie se encontraría en proceso activo de madurez a partir de los $35 \mathrm{~cm} \mathrm{LH.} \mathrm{A}$ pesar que bajo los $35 \mathrm{~cm}$ LH no se clasificó ninguna hembra como madura (Fig. 5), es probable que bajo esta longitud se puedan identificar hembras maduras empleando técnicas histológicas para determinar el estado de madurez gonadal. Esto se refleja en la asimetría que presenta el IC hacia un valor inferior de la TM (Fig. 5). El valor más probable para este parámetro, debería estar entonces, en torno a los $35 \mathrm{~cm}$ LH en las hembras.

Por otro lado, la TM $(36,9 \mathrm{~cm} \mathrm{LH})$ refleja una situación aceptable del régimen de operación de la flota pesquera sobre este recurso en la zona de pesca estudiada, ya que las capturas se sustentaron principalmente en ejemplares sobre los $39 \mathrm{~cm} \mathrm{LH,} \mathrm{lo}$ que estaría dando lugar a que los individuos participen al menos, en un evento reproductivo antes de ser capturados. De este modo la TM de B. australis, estaría siendo protegida con el régimen actual de operación de la flota pesquera artesanal del puerto de Lebu.

\section{AGRADECIMIENTOS}

El primer autor agradece al Gobierno Regional de la Región de Bío-Bío que otorgó la Beca que permitió el financiamiento parcial de este estudio. Agradece además a sus colegas Gustavo Aedo y Rodrigo Wiff por su colaboración en el análisis de los datos y a Santiago Gacitúa por el apoyo logístico. Especial reconocimiento a los revisores anónimos, quienes con sus correcciones y valiosos comentarios, ayudaron a mejorar el manuscrito.

\section{REFERENCIAS}

Alarcón, R. \& H. Arancibia. 1993. Talla de primera madurez sexual y fecundidad parcial en la merluza común, Merluccius gayi gayi (Guichenot, 1848). Cienc. Tecnol. Mar, 16: 33-45.

Arancibia, H., C. Cubillos, J. Remmaggi \& R. Alarcón. 1994. Determinación de la talla de primera madurez sexual y fecundidad parcial en la sardina común, Strangomera bentinki (Norman, 1936), del área de Talcahuano. Biol. Pesq., 23: 1117.

Arcos, D., S. Núñez \& A. Acuña. 1996. Variabilidad de pequeña escala en la zona nerítica del sistema de surgencia de Talcahuano (Chile Central): identificación y dinámica de áreas de retención larval. Gayana Oceanología, 4(1): 21-58.

Castro, L., G. Salinas \& E. Hernández. 2000. Environmental influences on winter spawning of the anchoveta Engraulis ringens off central Chile. Mar. Ecol. Progr. Ser., 197: 247-258.

Cubillos, L., R. Alarcón \& A. Brante. 1999. Empirical estimates of mortality for the Chilean hake (Merluccius gayi): evaluation of precision. Fish. Res., 42: 147-153.

Holden, M. \& D. Raitt. 1975. Manual de Ciencia Pesquera. Parte 2. Métodos para investigar los recursos y su aplicación. FAO, Doc. Téc. Pesca, 115: $1-211$.

Nikolsky, G.V. 1963. The ecology of fishes. Department of Ichthyology, Biology-Soil Faculty Moscow State University, 352 pp.

Oyarzún, C. 2001. Catálogo de los peces presentes en el sistema de corrientes de Humboldt frente a Chile Centro-Sur. Departamento de Oceanografía. 
Sección Pesquerías. Universidad de Concepción, Chile, 159 pp.

Pardo, A. \& J. Oliva. 1992. Estimación de talla de primera madurez sexual de caballa (Scomber japonicus peruanus) en la zona norte de Chile durante el período de máxima actividad reproductiva. Invest. Pesq., Chile, 37: 97-106.

Pavez, P., H. Cerisola, C. González \& C. Hurtado. 1998. Análisis y evaluación de la pesquería de la reineta (Brama australis) en el litoral de la V Región. Informe Final Proyecto FIP 96-26: 125 pp.

Parrish, R., C. Nelson \& A. Bakun. 1981. Transport mechanisms and reproductive success of fishes in the California Current. Bio1. Oceonogr., 1(2): 175203.

Pavlov, Yu. P. 1991a. Brama australis ValenciennesA valid species of sea bream (Bramidae) from southeastern Pacific Ocean. J. Ichthyol., 31(5): 6-9.

Recibido: 7 octubre 2002; Aceptado: 5 agosto 2003
Pavlov, Yu. P. 1991b. Information on morphometric and ecology of pomfret of the genus Brama inhabiting the southeastern Pacific Ocean. J. Ichthyol., 31(2): 120-124.

Roa, R., B. Ernst \& F. Tapia. 1999. Estimation of size at maturity: an evaluation of analytical and resampling procedures. US Fish. Bull., 97: 570580 .

Servicio Nacional de Pesca. 2001. Anuario Estadístico de Pesca Chile. (http://www.sernapesca.cl). Ministerio de Economía, Fomento y Reconstrucción. Servicio Nacional de Pesca, Santiago, Chile.

William, G., P. Joseph \& M. Mary. 1993. Biology of the Pacific pomfret (Brama japonica) in the Pacific Ocean. Can. J. Fish. Aquat. Sci., 50: 2608-2625. 